University of Nebraska - Lincoln

DigitalCommons@University of Nebraska - Lincoln

2019

Skew-symmetric splitting of high-order central schemes with nonlinear filters for computational aeroacoustics turbulence with shocks

Bjorn Sjögreen

Helen C. Yee

Alan A. Wray

Follow this and additional works at: https://digitalcommons.unl.edu/nasapub

Part of the Astrophysics and Astronomy Commons

This Article is brought to you for free and open access by the National Aeronautics and Space Administration at DigitalCommons@University of Nebraska - Lincoln. It has been accepted for inclusion in NASA Publications by an authorized administrator of DigitalCommons@University of Nebraska - Lincoln. 


\title{
Skew-symmetric splitting of high-order central schemes with nonlinear filters for computational aeroacoustics turbulence with shocks
}

\author{
B. Sjögreen ${ }^{1} \cdot$ H. C. Yee ${ }^{2}$ A. A. Wray²
}

Received: 19 October 2018 / Revised: 12 September 2019 / Accepted: 18 September 2019 / Published online: 10 October 2019

(c) This is a U.S. government work and not under copyright protection in the U.S.; foreign copyright protection may apply 2019

\begin{abstract}
A class of high-order nonlinear filter schemes by Yee et al. (J Comput Phys 150:199-238, 1999), Sjögreen and Yee (J Comput Phys 225:910-934, 2007), and Kotov et al. (Commun Comput Phys 19:273-300, 2016; J Comput Phys 307:189-202, 2016) is examined for long-time integrations of computational aeroacoustics (CAA) turbulence applications. This class of schemes was designed for an improved nonlinear stability and accuracy for long-time integration of compressible direct numerical simulation and large eddy simulation computations for both shock-free turbulence and turbulence with shocks. They are based on the skew-symmetric splitting version of the high-order central base scheme in conjunction with adaptive low-dissipation control via a nonlinear filter step to help with stability and accuracy capturing at shock-free regions as well as in the vicinity of discontinuities. The central dispersion-relation-preserving schemes as well as classical central schemes of arbitrary orders fit into the framework of skew-symmetric splitting of the inviscid flux derivatives. Numerical experiments on CAA turbulence test cases are validated.
\end{abstract}

Keywords High-order methods · CAA turbulence with shocks · Skew-symmetric splitting $\cdot$ High-order nonlinear filter $\cdot$ DRP schemes with nonlinear filter

\section{Introduction}

Improving nonlinear stability without smearing physical turbulent fluctuations for long-time integrations of turbulent flows with discontinuities poses a difficult challenge. Since the turn of this century, many optimized compact and non-compact weighted essentially non-oscillatory (WENO) and essentially non-oscillatory (ENO) schemes have been developed to reduce numerical dissipation away from discontinuity regions. See, e.g., [1-3]. These numerical methods are very high in CPU operation count and most often still

Communicated by C.-H. Chang.

$凶$ H. C. Yee

Helen.M.Yee@nasa.gov

B. Sjögreen

bjorn.sjogreen@multid.se

A. A. Wray

Alan.A.Wray@nasa.gov

1 Multid Analyses AB, Gothenburg, Sweden

2 NASA Ames Research Center, Mountain View, USA suffer from numerical stability/accuracy for long-time integration. Other numerical methods for combating excess numerical dissipation switch between non-dissipative (or low dissipative) spatial schemes and high-order high-resolution shock-capturing schemes. The blending of these two types of schemes requires extreme care to ensure numerical conservation and stability at interface locations [4]. More efficient numerical methods which avoid the interfacing problem are the nonlinear filter schemes [5-9]. Numerical stability can be improved by skew-symmetric splitting of the inviscid flux derivatives [10-12] and by high-order stable entropyconservative numerical fluxes $[13,14]$. Other sources of accuracy improvement are the dispersion-relation-preserving (DRP) schemes for computational aeroacoustics (CAA) [15] in conjunction with proper shock-capturing mechanisms.

The Yee and Sjögreen [16] adaptive nonlinear filter method consists of a high-order non-dissipative spatial base scheme and a nonlinear filter step. The nonlinear filter step consists of a flow sensor and the dissipative portion of a highresolution high-order shock-capturing method to guide the application of the shock-capturing dissipation where needed. The nonlinear filter idea was first initiated by Yee et al. [5] 
using an artificial compression method of Harten [17] as the flow sensor. Smart flow sensors were developed at a later stage by the same investigators and collaborators in [6-9]. The smart flow sensor provides the locations and the estimated strength of the necessary numerical dissipation needed at these locations and leaves the rest of the flow field free of shock-capturing dissipation. It is noted that the nonlinear filter approach of Yee and Sjögreen [16] requires one Riemann solve per time step per grid point for each spatial direction. It is independent of the time discretization to be used. However, hybrid schemes (switching between high-order non-dissipative methods and high-order shockcapturing methods) would require four Riemann solves per time step per grid point for each spatial direction if a fourthstage Runge-Kutta time discretization is used. Unlike the hybrid method, our highly parallelizable adaptive nonlinear filter schemes do not rely on switching between schemes to avoid the related numerical instability and conservation consideration at switching locations. These nonlinear filter schemes with adaptive numerical dissipation control in highorder shock-capturing schemes and their hybrid cousins have shown an excellent performance for certain turbulent test cases. For more practical 3D test cases of direct numerical simulation (DNS) and large eddy simulation (LES) of compressible shock-free turbulence, low-speed turbulence with shocklets, and supersonic turbulence for non-periodic boundaries in curvilinear geometries, some improvement in numerical stability is needed without resorting to added numerical dissipation that can interfere with the accuracy of numerical simulations.

In the early 1980s, skew-symmetric splitting of certain components of the inviscid flux derivatives in conjunction with central schemes was shown to help with numerical stability for long-time integration. For certain splittings, they can provide a stable energy norm estimate for the Euler equations with smooth flows. For other skew-symmetric formulations, they can provide a discrete momentum conservation or a discrete kinetic preservation property. See Yee and Sjögreen, Kotov et al., Yee et al., Yee and Sjögreen, Arakawa, Blaisdell et al., and Ducros [7-10,16,18-21] for some discussions and performance of the combined approach for DNS and LES applications. A semi-conservative skewsymmetric splitting (entropy splitting) of Yee et al. [10] in conjunction with the nonlinear filter approach to improve numerical stability without added ad hoc numerical dissipation was conducted in 2000. It has been utilized extensively in DNS of shock-free turbulence. See [22] and their later work for their wide applications. For their skew-symmetric splitting extension to the equations of ideal magnetohydrodynamics (MHD), see Yee et al., and Sjögreen et al. [10,11].

Traditionally, for shock-free CAA flows, formal order of accuracy of the scheme is not as important as reducing dispersion error in both time and space. Thus, the CAA community would rather use a lower-order DRP (dispersion relation preserving) scheme with a wider grid stencil than its standard central scheme counterpart. Most optimized DRP schemes prioritize optimizing the wave number at the expense of a wider grid stencil with the lower formal order (accuracy) than standard central schemes. On the one hand, acoustic waves interacting with shocks and turbulence-induced noise DRP schemes with linear filters alone usually are not capable of simulating such flows. As indicated in [23], a combination of a DRP linear filter and a shock-capturing nonlinear filter is needed. On the other hand, high-order compact WENO schemes, e.g., [3] and references cited therein, are more CPU intensive but exhibit no obvious improvement for long-time integration of compressible shock-free turbulence and turbulence with shocks than the high-order nonlinear filter approach (or their hybrid method counterparts) $[8,9,15,16,24,25]$. Due to this fact, here, the Yee and Sjögreen nonlinear filter step with shock-capturing properties is designed to replace the spatial DRP linear filter for CAA turbulence with shock waves. In addition, we consider the Ducros et al. two-factor and Kennedy-Gruber three-factor conservative skew-symmetric splittings $[20,26]$ as part of the Yee and Sjögreen [16] nonlinear filter scheme framework to improve nonlinear stability for long-time integration. Both the classical high-order central schemes and central DRP schemes as part of the base schemes are considered. The Ducros et al. splitting will henceforth be referred to as DS.

This paper only considers high-order spatial discretizations by the method-of-lines approach. Appropriate, accurate, stable time discretization is assumed. Development or utilization of time discretization schemes is beyond the scope of the current investigation. This is a sequel to $[15,24]$ with application to CAA turbulence with shocks. After a brief introduction and motivation, the skew-symmetric splitting form for both high-order central schemes and the central DRP schemes will be briefly reviewed. Then, for low-dissipative high-order shock-capturing methods that were designed for long-time integration of turbulent flows with discontinuities, our most updated nonlinear filter approach will be briefly described. Grid refinement studies for two CAA test cases with shocks comparing different high-order nonlinear filter methods will be included. Due to our adaptive flow sensor to control the location and amount of numerical dissipation where needed, the dissipative portion of the high-order shock-capturing scheme is only utilized at isolated computational regions, while maintaining high accuracy almost everywhere. Our numerical experiments only considered the less-CPU-intensive dissipative portion of high-resolution shock-capturing schemes as candidates for our nonlinear filter approach. Although the dissipative portion of optimized high-order WENO, ENO, compact WENO, or another moreCPU-intensive high-resolution shock-capturing scheme fits into the framework of our nonlinear filter scheme frame- 
work, due to the CPU-intensive nature of these schemes, they are not considered in our numerical experiments. In addition, shock-capturing schemes that concentrate on capturing discontinuities with a narrowest grid stencil are usually designed for the rapidly developing flows. Often, for a longtime integration of DNS and LES types of flows, other added mechanisms are needed to improve nonlinear stability.

\section{An overview of skew-symmetric split approximations for gas dynamics}

Standard spatial centered difference approximations of nonlinear conservation laws normally encounter nonlinear instabilities after a short-time integration without added numerical dissipation. It is well known that the appearance of these instabilities can be delayed if the convective flux derivatives are written in an equivalent desired split form before the pure central approximation is employed. Hereafter, this is referred to as a split approximation.

For example, a split approximation starts from rewriting the derivative of the product $(a b)_{x}$ as

$(a b)_{x}=\alpha(a b)_{x}+\gamma a b_{x}+\beta a_{x} b$

before discretization. Here, $a$ and $b$ are functions of $x$ and $\alpha, \gamma$, and $\beta$ are parameters chosen to be still equivalent to the original $(a b)_{x}$ before discretization. A common split derivative is found by setting $\alpha=\gamma=\beta=1 / 2$ resulting in the form

$(a b)_{x}=\frac{1}{2}(a b)_{x}+\frac{1}{2} a b_{x}+\frac{1}{2} a_{x} b$.

As another example, a split approximation for the derivative of the triple product of three functions $(a b c)_{x}$ is

$$
\begin{aligned}
(a b)_{x}= & \alpha(a b c)_{x}+\gamma\left[a(b c)_{x}+b c a_{x}\right]+\beta\left[b(a c)_{x}+a c b_{x}\right] \\
& +\kappa\left[c(a b)_{x}+a b c_{x}\right]+\delta\left[b c a_{x}+a c b_{x}+a b c_{x}\right]
\end{aligned}
$$

before discretization. Here, $a, b$, and $c$ are functions of $x$ and $\alpha, \gamma, \beta, \kappa$, and $\delta$ are parameters chosen to be still equivalent to the original $(a b c)_{x}$ before discretization.

A common parameter choice was established by Kennedy and Gruber, and Pirozzoli $[26,27]$ for the nonlinear Euler equation by setting $\alpha=\gamma=\beta=\kappa=1 / 4$ and $\delta=0$ for the triple-product derivative. The resulting splitting in conjunction with the central scheme is kinetic energy preserving in the discretized sense.

The next section briefly derives the Ducros et al. [20]-type conservative splitting (DS) for $2 p$ th-order central schemes. For the corresponding numerical fluxes in the DS split form for the nonlinear Euler and MHD equations, see $[10,11,15]$. A similar procedure can be derived for the Kennedy-Gruber splitting of arbitrary even order for central schemes. A comparison of these skew-symmetric splittings can be found in [28] and its extended journal version.

\subsection{Ducros et al.-type conservative splitting (DS)}

For nonlinear systems, such as the Euler equations of gas dynamics, split approximations have been used for a long time; see, e.g., Blaisdell et al. and Ducros et al. [19,20]. The Ducros et al. split approximation starts with (2) with the terms of the split form approximated by

$$
\frac{1}{2} D(a b)+\frac{1}{2} D(a) b+\frac{1}{2} a D(b),
$$

where $D$ is a centered finite difference operator and $a$ and $b$ are functions of $x$.

The key step in the DS [20] split approximation is to rewrite (4) in the conservation form. For example, with second-order operator $D u_{j}=\left(u_{j+1}-u_{j-1}\right) /(2 \Delta x)$, it holds that

$$
\begin{aligned}
& \frac{1}{2} D(a b)+\frac{1}{2} D(a) b+\frac{1}{2} a D(b) \\
& \quad=\frac{1}{4 \Delta x} \Delta_{+}\left[\left(a_{j}+a_{j-1}\right)\left(b_{j}+b_{j-1}\right)\right],
\end{aligned}
$$

where $\Delta_{+} q_{j}=\left(q_{j+1}-q_{j}\right)$.

Equation (5) can be generalized to standard centered difference operators of $2 p$ th-order of accuracy,

$D_{p} u_{j}=\frac{1}{\Delta x} \sum_{k=1}^{p} \alpha_{k}^{(p)}\left(u_{j+k}-u_{j-k}\right)$

The coefficients $\alpha_{k}^{(p)}$ satisfy

$$
\sum_{k=1}^{p} k \alpha_{k}^{(p)}=\frac{1}{2} \quad \sum_{k=1}^{p} \alpha_{k}^{(p)} k^{2 n+1}=0, n=1, \ldots, p-1 .
$$

To derive the conservative form of the split approximation for an arbitrary operator, the right-hand side of the algebraic identity

$$
\begin{aligned}
& a_{j+k} b_{j+k}-a_{j-k} b_{j-k}+\left(a_{j+k}-a_{j-k}\right) b_{j}+a_{j}\left(b_{j+k}-b_{j-k}\right) \\
& =\left(a_{j+k}+a_{j}\right)\left(b_{j+k}+b_{j}\right)-\left(a_{j}+a_{j-k}\right)\left(b_{j}+b_{j-k}\right)
\end{aligned}
$$


is written in the conservation form by

$$
\begin{aligned}
& \left(a_{j+k}+a_{j}\right)\left(b_{j+k}+b_{j}\right)-\left(a_{j}+a_{j-k}\right)\left(b_{j}+b_{j-k}\right) \\
& =\sum_{m=0}^{k-1}\left(a_{j-m}+a_{j+k-m}\right)\left(b_{j-m}+b_{j+k-m}\right) \\
& \quad-\sum_{m=0}^{k-1}\left(a_{j-1-m}+a_{j-1+k-m}\right)\left(b_{j-1-m}+b_{j-1+k-m}\right) .
\end{aligned}
$$

The conservative form of the split approximation becomes

$$
\begin{aligned}
& \frac{1}{2} D_{p}(a b)+\frac{1}{2} D_{p}(a) b+\frac{1}{2} a D_{p}(b) \\
& =\frac{1}{\Delta x} \sum_{k=1}^{p} \frac{1}{2} \alpha_{k}^{(p)}\left(\left(a_{j+k} b_{j+k}-a_{j-k} b_{j-k}\right)\right. \\
& \left.\quad+a_{j}\left(b_{j+k}-b_{j-k}\right)+\left(a_{j+k}-a_{j-k}\right) b_{j}\right) \\
& =\frac{1}{\Delta x} \sum_{k=1}^{p} \frac{\alpha_{k}^{(p)}}{2}\left(\sum_{m=0}^{k-1}\left(a_{j-m}+a_{j+k-m}\right)\left(b_{j-m}+b_{j+k-m}\right)\right. \\
& \left.\quad-\sum_{m=0}^{k-1}\left(a_{j-1-m}+a_{j-1+k-m}\right)\left(b_{j-1-m}+b_{j-1+k-m}\right)\right) \\
& =\frac{1}{\Delta x}\left(h_{j+1 / 2}-h_{j-1 / 2}\right),
\end{aligned}
$$

where the numerical flux is defined by

$$
h_{j+1 / 2}=\sum_{k=1}^{p} \frac{1}{2} \alpha_{k}^{(p)} \sum_{m=0}^{k-1}\left(a_{j-m}+a_{j+k-m}\right)\left(b_{j-m}+b_{j+k-m}\right) .
$$

\section{DRP schemes}

In the turbulence and CAA communities, researchers prefer to utilize wave-number-optimized schemes for general DNS and LES applications. In this study, two different optimized finite difference operators are considered to compare with the high-order classical central scheme. See Tam [29] and De Roeck et al. [30] for the development and references cited therein. These are: (a) DRP4S7, the original Tam and Webb fourth-order accurate DRP operator with a seven-point wide grid stencil, and (b) DRP4S9, the fourth-order accurate DRP operator with a nine-point wide grid stencil. Both operators have antisymmetric coefficients and are optimized over wave number intervals: $0 \leq k \Delta x \leq 1.1$ for DRP4S7 and $\pi / 16 \leq$ $k \Delta x \leq \pi / 2$ for DRP4S9. Here, $\Delta x$ is the grid spacing and the integer $k$ is the mode number. DRP4S7 was studied in [30].

Remark Numerical experiments made with DRP4S7 optimized over $\pi / 16 \leq k \Delta x \leq \pi / 2$ gave worse accuracy than with DRP4S7 optimized over the more standard choice
Table 1 Coefficients of DRP4S7, optimized over $[0,1.1]$

Table 2 Coefficients of DRP4S9, optimized over $[\pi / 16, \pi / 2]$

$0 \leq k \Delta x \leq 1.1$ used here. It is reasonable to expect that with fewer free parameters, the interval of optimization would be made shorter. DRP4S7 and DRP4S9 use least square minimization of the absolute error, i.e., integral over the square of the error in wave number space.

Their difference operators $D$ for the first-order derivative of a grid function $u_{j}$ are of the form

$D u_{j}=\frac{1}{\Delta x} \sum_{k=1}^{q} a_{k}\left(u_{j+k}-u_{j-k}\right)$.

Table 1 gives the coefficients of the DRP4S7 scheme. Table 2 lists the coefficients of the DRP4S9 scheme.

Note that the centered operators (12) are of the same antisymmetric form as (6). This implies that the DS splitting and the Kennedy-Gruber splitting described in the previous subsection are also straightforwardly applicable to be used for the optimized operators described in this subsection.

\section{Skew-symmetric split form of classical central and DRP differencing as base schemes in the framework of the nonlinear filter method of Yee and Sjögreen, Kotov et al., and Yee and Sjögreen [7-9,12,16]}

Before the application of a high-order non-dissipative central spatial base scheme, a preprocessing step is employed to improve numerical stability. In the turbulence community, it is often referred to as de-aliasing errors. The inviscid flux derivatives of the governing equations are split into the following two ways, depending on the flow types and the desire for rigorous mathematical analysis or physical argument.

- Entropy splitting of [10] or the natural splitting [21]: These are non-conservative splittings, and they are among some of the best in improving the numerical sta- 
bility for non-dissipative central schemes, especially for long-time integration of shock-free turbulence.

- The DS splitting [20] and Kennedy-Gruber splitting $[26,27]$ for systems: These are conservative splittings and are suitable for problems with discontinuities.

Remark For problems containing discontinuities, conservative skew-symmetric splittings should be used.

\subsection{Base scheme step after the preprocessing step}

A full time step is advanced using a high-order nondissipative (or very low dissipation) spatially central scheme approximating the split form of the governing partial differential equations (PDEs) (i.e., after the preprocessing step). For the current study, sixth-order to eighth-order classical central schemes and the DRP4S7 and DRP4S9 DRP schemes are considered as base schemes.

For the base scheme step, a full time step of high-order temporal discretization such as the third- and fourth-order Runge-Kutta (RK3 and RK4) method is used. Other DRP temporal discretizations could be used for the base scheme step, but are beyond the scope of this investigation. See Tam [29,31], Brambley [32], and Haras and Ta'asan [33].

\subsection{Post-processing (nonlinear filter step)}

To further improve the accuracy of the computed solution from the base scheme step, after a full time step of a nondissipative high-order spatial base scheme on the split form of the governing equation(s), the post-processing step nonlinearly filters the solution by a dissipative portion of a high-order shock-capturing scheme with a local flow sensor. A comparable order of accuracy of the nonlinear filter dissipation with the base scheme is usually considered. For non-entropy-satisfying shock-capturing schemes, it is assumed that entropy-satisfying fixes for both 1D and multi$\mathrm{D}$ are employed as indicated in Yee et al. [34]. For extreme flows, positivity-preserving shock-capturing schemes should be used. See Kotov et al. [35] for some performance of positivity-preserving nonlinear filter schemes.

The flow sensor provides locations and amounts of builtin shock-capturing dissipation that can be further reduced or eliminated. At each grid point, a local flow sensor is employed to analyze the regularity of the computed flow data. Only the strong discontinuity locations would receive the full amount of shock-capturing dissipation. In smooth regions, no shock-capturing dissipation would be added unless highfrequency oscillations develop, owing to the possibility of numerical instability in long-time integrations of nonlinear governing PDEs. In regions with strong turbulence, if needed, a small fraction of the shock-capturing dissipation would be added to improve stability.
In one space dimension, the numerical flux function used in the nonlinear filter step is described by the formula

$h_{j+1 / 2}^{(\mathrm{f})}=s_{j+1 / 2}\left(h_{j+1 / 2}^{(\text {weno }}-h_{j+1 / 2}^{(\mathrm{c})}\right)$,

where $s_{j+1 / 2}$ is the flow sensor, $h_{j+1 / 2}^{\text {(weno) }}$ is a WENO numerical flux function, and $h_{j+1 / 2}^{(\mathrm{c})}$ is a centered numerical flux. The $2 p$ th-order accurate centered flux is used together with the $2 p-1$ accurate WENO flux. The wavelet flow sensor consists of two factors

$s_{j+1 / 2}=\kappa w_{j+1 / 2}$

where $\kappa$ is a constant and $w_{j+1 / 2} \in[0,1]$ is a normalized function of the local Lipschitz exponent of the flow field, estimated from the wavelet coefficients, see [6] for details. The numerical experiments in this paper were made with a threelevel wavelet sensor, based on the fourth-order redundant B-spline wavelet basis. In the 2D shock-vortex interaction problem, the Lipschitz cutoff exponent was set to $\alpha_{0}=0.5$ and the premultiplying factor $\kappa=0.5$. In the duct flow problem, the parameters were set to $\alpha_{0}=0.9$ and $\kappa=1$. In both cases, the LLF variant of the WENO scheme was used.

\section{Numerical experiments}

To validate our high-order nonlinear filter approach, a $2 \mathrm{D}$ inviscid stationary shock interacting with a pair of counterrotating vortices [36] and a 2D inviscid supersonic flow entering a duct through a nozzle [23] are considered. A large number of computations using different levels of grid refinement for each test case were made. The finest grid acts as the reference solution to compare the accuracy of the studied schemes.

\subsection{Test Case 1: 2D inviscid shock-vortex interaction}

This is a 2D inviscid test problem reported in [36] where two vortices pass through a steady shock wave. The constant states on the left (subscript L) and on the right (subscript R) of the steady shock wave are

$(\rho, u, v, p)_{\mathrm{L}}=(1.3416,-0.8944,0,1.0810)$

$(\rho, u, v, p)_{\mathrm{R}}=(1,-1.2,0,1 / \gamma)$,

where $\gamma=1.4$. This makes the speeds of sound $c_{\mathrm{L}}=1.0621$ and $c_{\mathrm{R}}=1$, and the Mach numbers $M_{\mathrm{L}}=0.8421$ and $M_{\mathrm{R}}=$ 1.2. Hence, the right state is supersonic, giving supersonic inflow conditions at the upstream (right) boundary. The left state is subsonic, giving subsonic outflow conditions at the downstream (left) boundary. The domain is square of size 
$-40 \leq x \leq 20,-30 \leq y \leq 30$. The shock wave is located at $x=0$. Here, all sizes are given in non-dimensional units.

Initially, the vortices are superimposed on the right-hand state with vortex one centered at $\left(x_{1}, y_{1}\right)$ and vortex two centered at $\left(x_{2}, y_{2}\right)$. The right state is modified to

$\rho_{\mathrm{R}}=\left(1-\frac{1}{2}(\gamma-1) \beta^{2} \mathrm{e}^{1-r_{1}^{2}}-\frac{1}{2}(\gamma-1) \beta^{2} \mathrm{e}^{1-r_{2}^{2}}\right)^{1 /(\gamma-1)}$

$u_{\mathrm{R}}=-1.2-\beta\left(y-y_{1}\right) \mathrm{e}^{\left(1-r_{1}^{2}\right) / 2}+\beta\left(y-y_{2}\right) \mathrm{e}^{\left(1-r_{2}^{2}\right) / 2}$

$v_{\mathrm{R}}=\beta\left(x-x_{1}\right) \mathrm{e}^{\left(1-r_{1}^{2}\right) / 2}-\beta\left(x-x_{2}\right) \mathrm{e}^{\left(1-r_{2}^{2}\right) / 2}$

$p_{\mathrm{R}}=\rho_{\mathrm{R}}^{\gamma} / \gamma$,

where the pressure definition makes the entropy $p \rho^{-\gamma}$ equal to $1 / \gamma$. The distances are defined by $r_{1}^{2}=\left(x-x_{1}\right)^{2}+$ $\left(y-y_{1}\right)^{2}$ and $r_{2}^{2}=\left(x-x_{2}\right)^{2}+\left(y-y_{2}\right)^{2}$. The vortex centers are $\left(x_{1}, y_{1}\right)=(4,-2 \cos \alpha)$ and $\left(x_{2}, y_{2}\right)=$ $(4+4 \sin \alpha, 2 \cos \alpha)$. The vortex strength, $\beta$, and vortex alignment, $\alpha$, are problem parameters.

In the example computations, $\beta=0.05$ and $\alpha=45^{\circ}$. The computations are run to time 15 . The plotted acoustic pressure is defined by $p_{\mathrm{a}}=\left(p-p_{0}\right) / p_{0}$, where $p_{0}=1.0810$ is the left state pressure. Figure 1 shows velocity magnitude, $\sqrt{u^{2}+v^{2}}$, and contours for the initial data.

Figures 2, 3, and 4 show the acoustic pressure contours at $t=15$ for three grids $(201 \times 201,401 \times 401$, and $801 \times 801$ ), comparing seventh-order WENO (WENO7) with the two central base schemes DRP4S9DS and C08 in conjunction with the dissipative portion of WENO7 (WENO7FI) as the nonlinear filter. DRP4S9DS indicates the use of the DS split form of DRP4S9. C08 + WENO7FI indicates the eighth-order classical central scheme in conjunction with

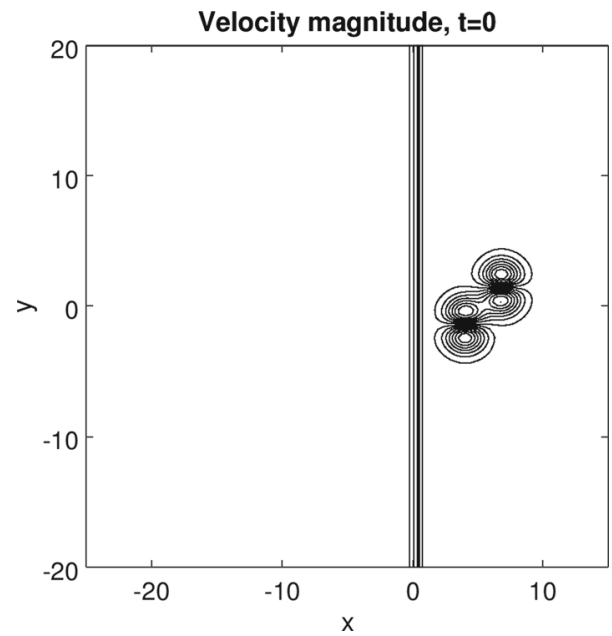

Fig. 1 Shock-vortex interaction: velocity magnitude of initial data with $\alpha=45^{\circ}$
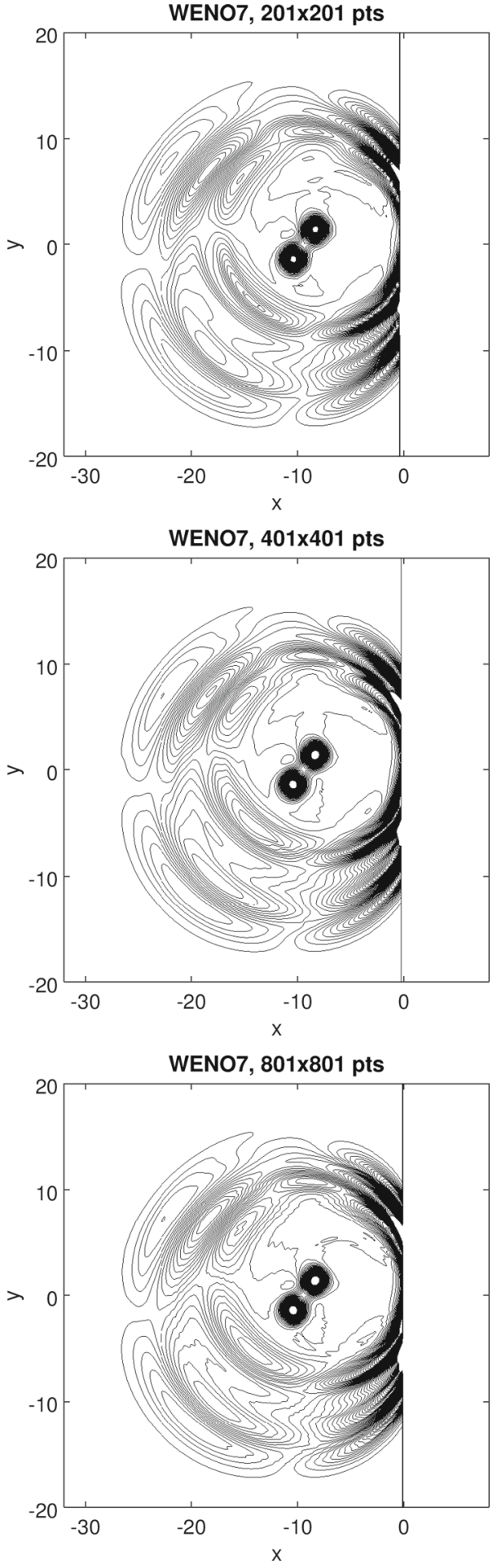

Fig. 2 Shock-vortex interaction: acoustic pressure contours at $t=15$. WENO7, $201 \times 201$ grid points (top), $401 \times 401$ grid points (middle), and $801 \times 801$ grid points (left)

the dissipative portion of WENO7 as the nonlinear filter. Figures 5, 6, and 7 show the corresponding contour line plots using a finer grid as the reference solution for the three schemes with and without the DS splitting. Results with 

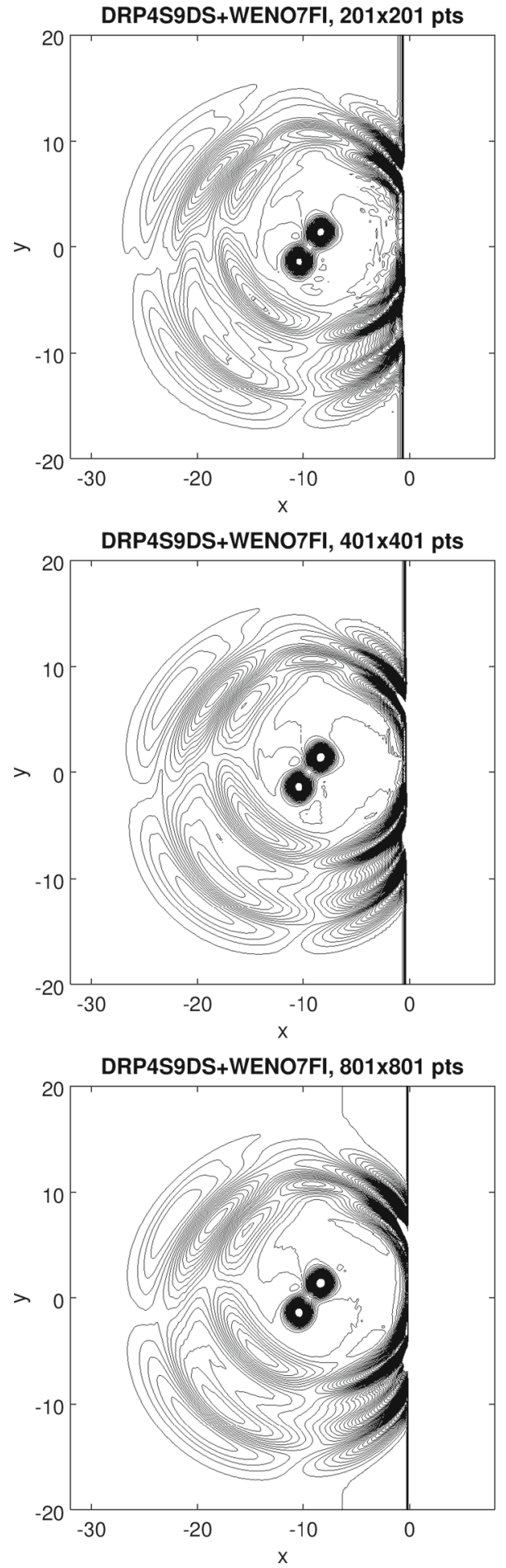

Fig. 3 Shock-vortex interaction: acoustic pressure contours at $t=15$. DRP4S9DS + WENO7FI, $201 \times 201$ grid points (top), $401 \times 401$ grid points (middle), and $801 \times 801$ grid points (bottom)

C08DS, the DS split form of C08, as the base scheme are also shown.

Figures 8 and 9 show acoustic pressure comparisons of two selected cross sections for the four-grid and five high-
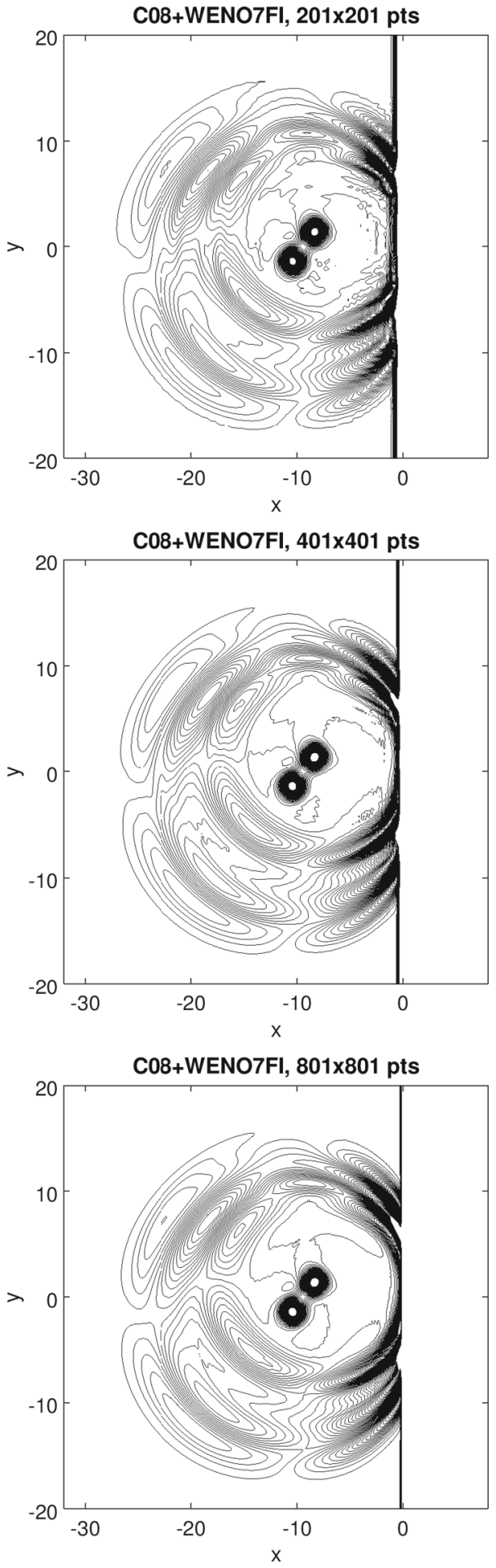

Fig. 4 Shock-vortex interaction: acoustic pressure contours at $t=15$. C08 + WENO7FI, $201 \times 201$ grid points (top), $401 \times 401$ grid points (middle), and $801 \times 801$ grid points (bottom)

order methods. There is a definite gain in accuracy by DRP4S9DS compared with DRP4S9 (it is less oscillatory with improved shock capturing; see top figure in Fig. 7 in the vicinity of the shock and Figs. 8 and 9 at the oscilla- 


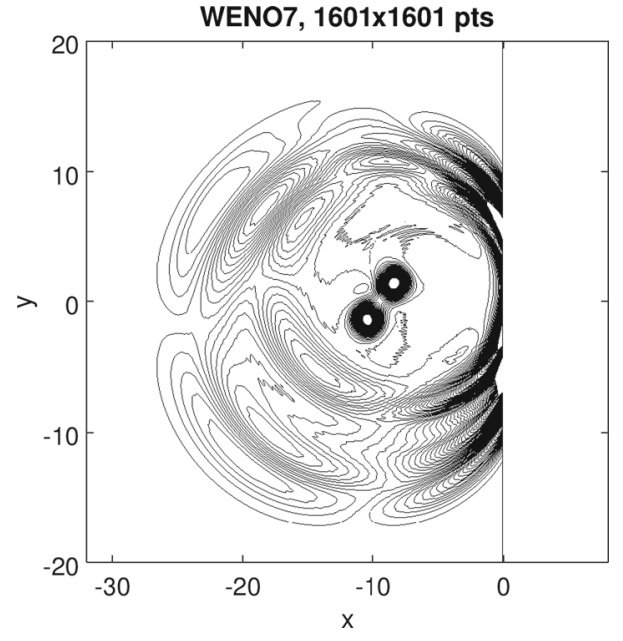

Fig. 5 Shock-vortex interaction: acoustic pressure contours at $t=15$. WENO7, $1601 \times 1601$ grid
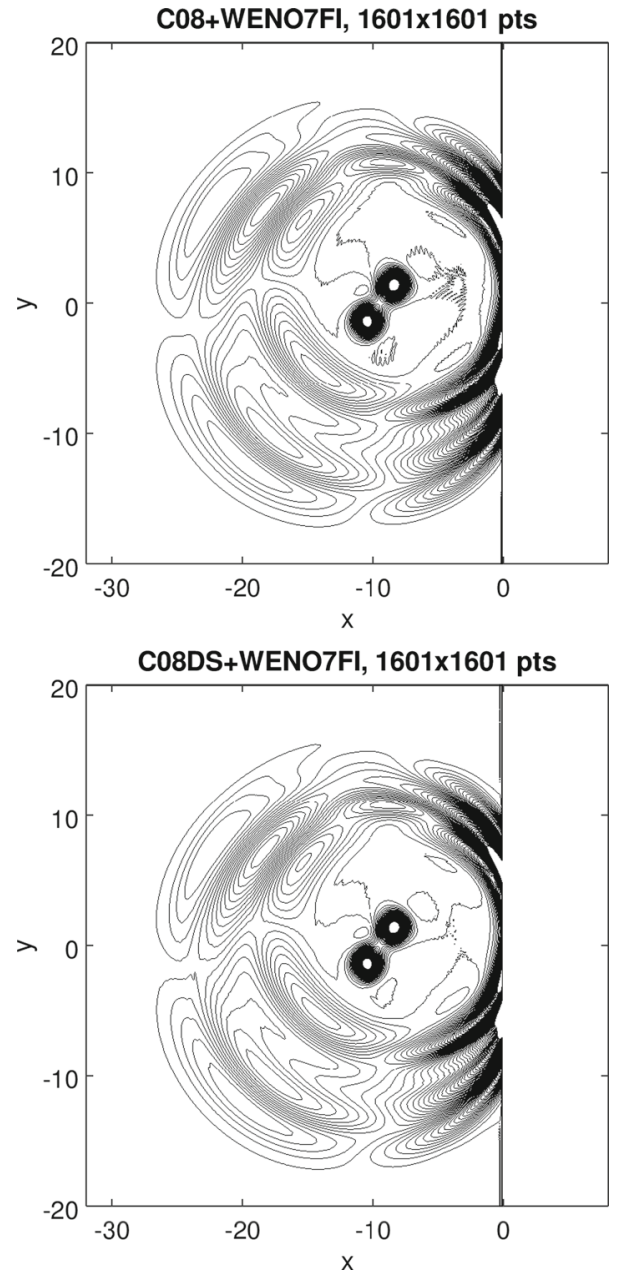

Fig. 6 Shock-vortex interaction: acoustic pressure contours at $t=15$. C08+WENO7FI (top) and C08DS + WENO7FI (bottom), $1601 \times 1601$ grid
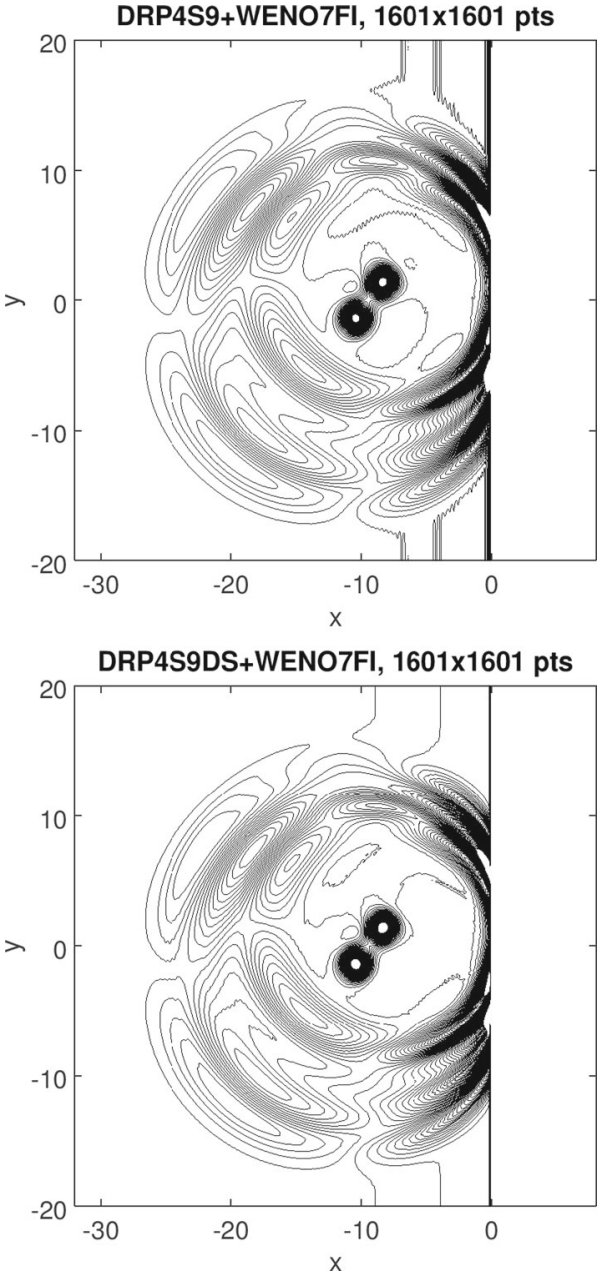

Fig. 7 Shock-vortex interaction: acoustic pressure contours at $t=15$. DRP4S9 + WENO7FI (top) and DRP4S9DS + WENO7FI (bottom), $1601 \times 1601$ grid

tory portion of the cross section curves). Figure 10 shows the solutions of five methods for the coarse grid $201 \times 201$ at selected cross sections compared with a reference solution obtained by WENO7 on a grid with $1601 \times 1601$ grid points. For C08 + WENO7FI versus C08DS + WENO7FI, there is less gain in accuracy with or without DS splitting. Although there is a minor gain in accuracy by the nonlinear filter scheme over the WENO scheme for this particular test case, in terms of efficiency, the nonlinear filter step only requires one Riemann solve per time step, regardless of the time discretization. For their WENO scheme counterparts or hybrid scheme variants, Riemann solvers are needed at every Runge-Kutta stage resulting in higher computational cost. Hybrid schemes here are the blending of two schemes with a flow sensor to switch between two schemes as described in Sect. 1. 

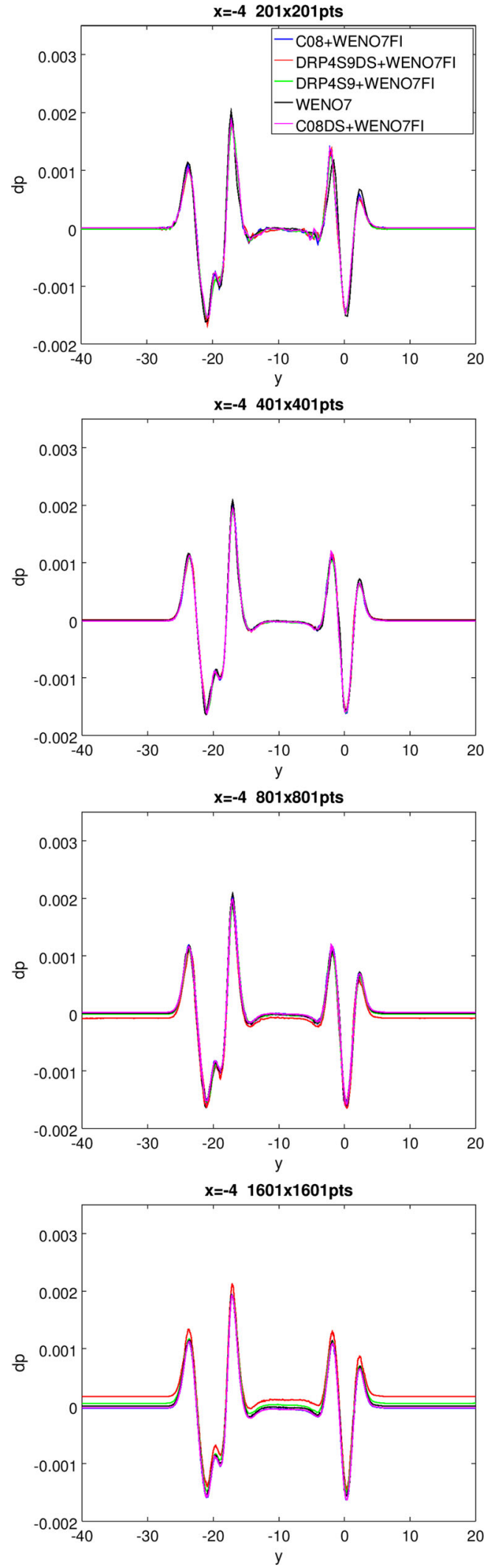

Fig. 8 Shock-vortex interaction: acoustic pressure at $x=-4$. Comparison of several schemes for four grids
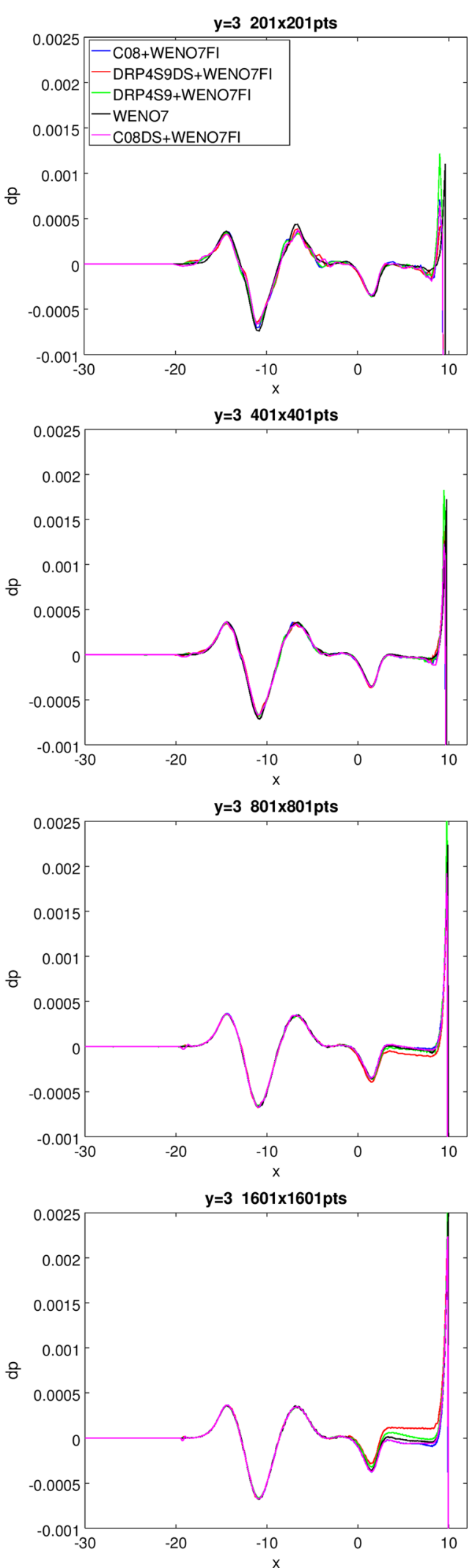

Fig. 9 Shock-vortex interaction: acoustic pressure at $y=3$. Comparison of several schemes for four grids 

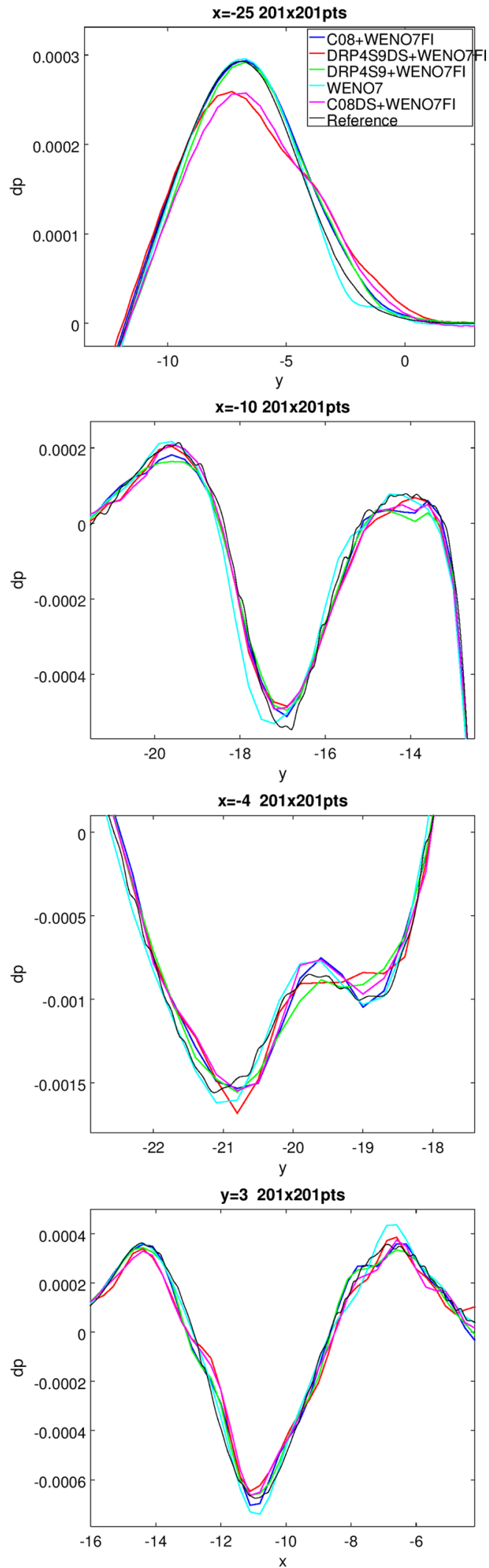

Fig. 10 Shock-vortex interaction: acoustic pressure at $x=-25$, $x=-10, x=-4$, and $y=3$, grid $201 \times 201$ compared with the reference grid solution

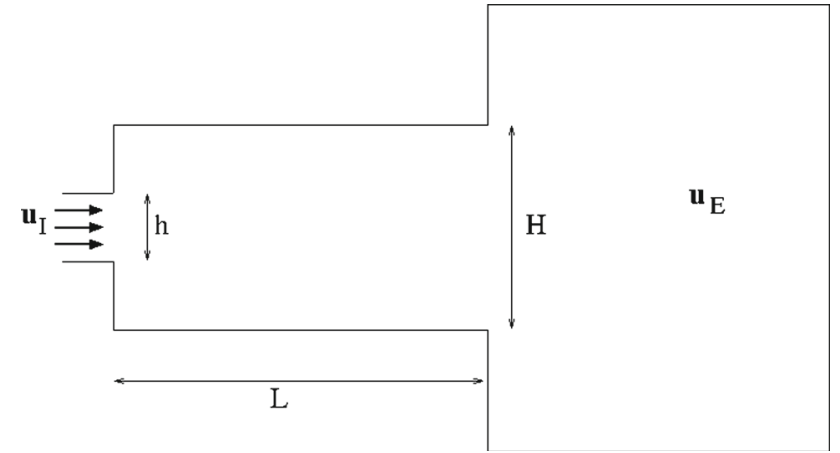

Fig. 11 Domain of the problem

\subsection{Test Case 2: duct flow}

The second test case is a 2D inviscid supersonic flow entering a duct through a nozzle. The flow exits the duct into a reservoir of the given pressure. The Navier-Stokes version of this problem was described in [23], where both computations and comparisons with experimental results were made for a pseudo-3D overset grid computation with a very fine grid discretizing the boundary-layer regions. As described in [23], the interest in this problem is to compute flows where acoustic duct modes interact with modes from the unsteady shock waves. The domain is outlined in Fig. 11. We follow [23] by using the height of the duct $H=0.033195 \mathrm{~m}$ as the unit of length in the geometrical description. The duct length is $L=7.23 H$, and the inflow nozzle height is $h=0.3 H$. In the study of Emmert et al., the 3D geometry is extended periodically in the direction normal to Fig. 11, with length $b=3.0125 H$.

The inflow state from the nozzle is imposed as a boundary condition on the left boundary of the duct. The fluid is a gamma-law gas with $\gamma=1.4$. The inflow state $\mathbf{u}_{\mathrm{I}}$ and reservoir state $\mathbf{u}_{\mathrm{E}}$ are given by

$$
\left(\begin{array}{l}
\rho_{\mathrm{I}} \\
u_{\mathrm{I}} \\
v_{\mathrm{I}} \\
p_{\mathrm{I}}
\end{array}\right)=\left(\begin{array}{c}
0.75818 \\
315.67 \\
0 \\
52902
\end{array}\right) \quad\left(\begin{array}{l}
\rho_{\mathrm{E}} \\
u_{\mathrm{E}} \\
v_{\mathrm{E}} \\
p_{\mathrm{E}}
\end{array}\right)=\left(\begin{array}{c}
1.2062 \tau \\
0 \\
0 \\
1.0123 \times 10^{5} \tau
\end{array}\right) \text {, }
$$

where the numbers are given in SI units. The inflow is supersonic with Mach number 1.01. The problem parameter $\tau$ influences the behavior of the flow. It has been observed experimentally that for low values of $\tau$, a regular steady shock cell pattern develops. As $\tau$ is increased, the shock structure breaks up and becomes unsteady; see [23]. The density is denoted by $\rho$, and $u, v$, and $w$ are the velocities in the $x-, y$-, and $z$-direction, respectively. The pressure is denoted by $p$. 


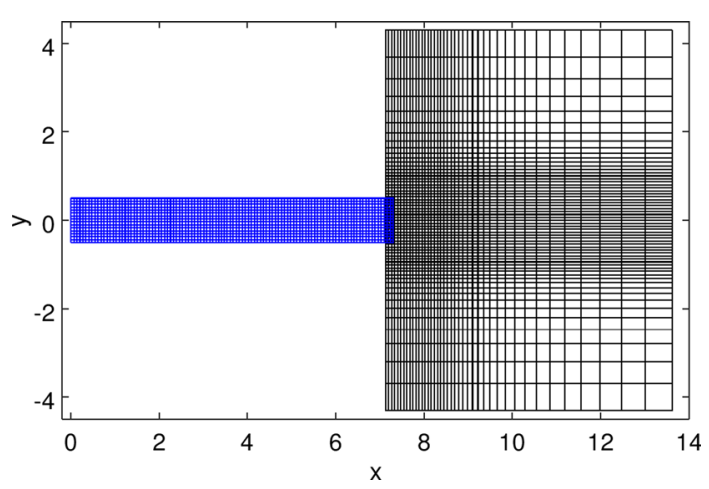

Fig. 12 Inviscid duct flow: uniform Cartesian duct grid with stretched reservoir grid

The viscosity is given by Sutherland's law

$\mu(T)=\mu\left(T_{\text {ref }}\right)\left(\frac{T}{T_{\text {ref }}}\right)^{1.5}\left(\frac{T_{\text {ref }}+C}{T+C}\right)$,

where $T_{\text {ref }}=273 \mathrm{~K}, C=110 \mathrm{~K}, \mu\left(T_{\text {ref }}\right)=1.5 \times$ $10^{-5} \mathrm{~kg} \mathrm{~m}^{-1} \mathrm{~s}^{-1}$. The Reynolds number based on the length scale $H$ and the inflow speed of sound $\sqrt{\gamma p_{\mathrm{I}} / \rho_{\mathrm{I}}}=$ $312.55 \mathrm{~m} \mathrm{~s}^{-1}$ as the velocity scale is $6.9167 \times 10^{5}$. With this scaling, the flow variables are all of unit order of magnitude. The Prandtl number is 0.72 .

In the discussion and plots below, the lengths $(x, y)$ are scaled by $H$, but all other quantities are given in SI units.

Adiabatic wall boundary conditions are imposed on the boundaries of the duct, except for the left boundary where the inflow state is imposed on $-0.15<y<0.15$.

In our study, the main goal is to show the performance of several high-order, shock-capturing schemes concentrating on the nonlinear Euler equations. Here, only the inviscid 2D flow counterpart of a symmetric-like steady supersonic flow case for $\tau=0.15$ of [23] is considered. We considered two overset grids for our study. One is the uniform Cartesian grid on the duct, and the other is the stretched Cartesian grid on the reservoir as shown in Fig. 12. At the right boundary of the duct, the reservoir and duct grids have the same spacing. This results in grid points of the two grids coinciding on the overlap, making it straightforward to apply interface conditions. The dimensions of the reservoir grid in computations are $47 \times 55$, corresponding to stretching the grid spacing at the far-field boundary to 100 times the grid spacing of the duct grid. Characteristic inflow/outflow boundary conditions are used on its sides. The grid is stretched to be coarser near the boundaries, as in a supergrid sponge layer.

The exit state is imposed in the entire domain initially. The evolution of the pressure in time is shown in Fig. 13. The figure shows pressure contour levels at five times up to $t=8.13 \times 10^{-3} \mathrm{~s}$. The flow pattern has not reached a quasisteady state. This computation used the WENO5 spatial
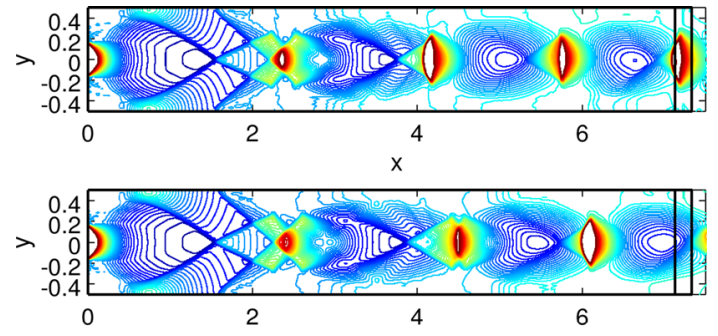

$x$

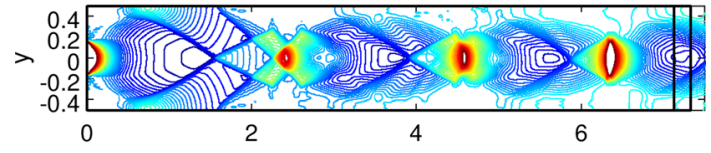

$\mathrm{x}$

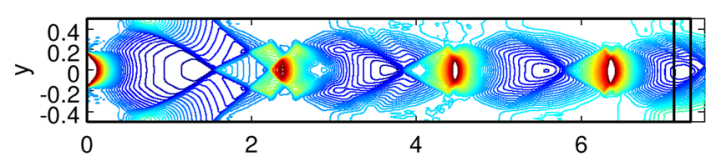

$x$

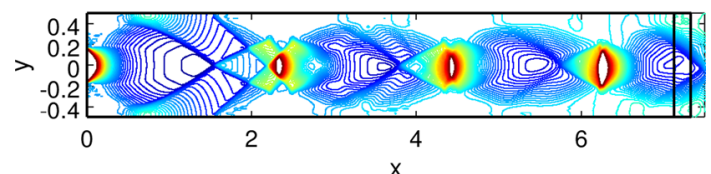

Fig. 13 Inviscid duct flow: pressure contours by WENO5 at times $t=3.48,4.65,5.81,6.97$, and $8.13 \mathrm{~ms}$, from top to bottom. Duct grid has $431 \times 61$ grid points

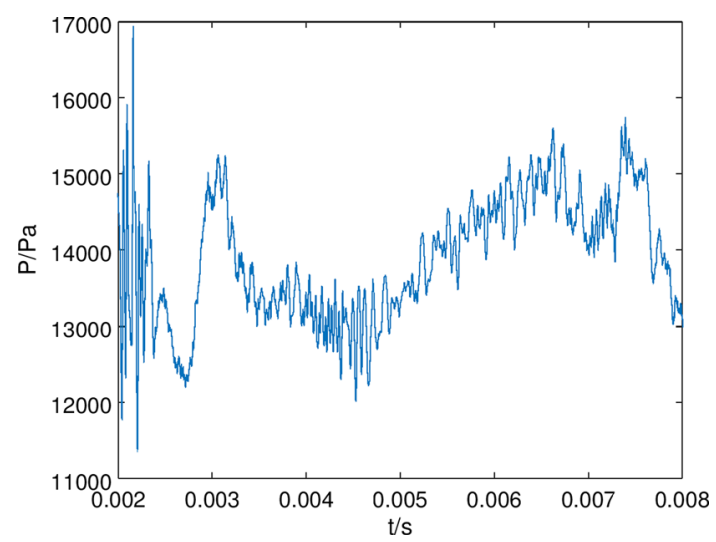

Fig. 14 Inviscid duct flow: pressure versus time by WENO5 at the upper wall of the duct at $x=3.615$

discretization with $431 \times 61$ grid points in the duct grid and $167 \times 241$ in the reservoir grid. A third-order Runge-Kutta method integrated the equations in time, using 36,000 time steps and CFL number 0.85 . Figure 14 shows the recorded pressure vs. time at the upper wall at the point $x=3.615$.

Due to the long-time integration nature of this test case, to gain a first-hand numerical study, only WENO5 and its nonlinear filter counterparts are considered. Figure 15 shows pressure contours at time $1.32 \mathrm{~ms}$, computed by three different schemes. The upper subplot shows the solution by the WENO5 scheme. The middle subplot shows the solution by the nonlinear filter C06DS+WENO5FI. The nonlinear fil- 


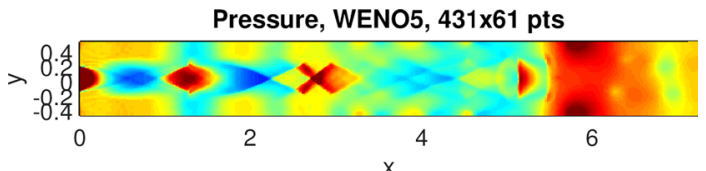

Pressure, C06DS+WENO5FI, 431 x61 pts

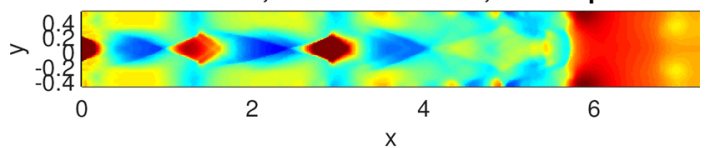

Pressure, DRP4S7+WENO5FI, 431 x61 pts

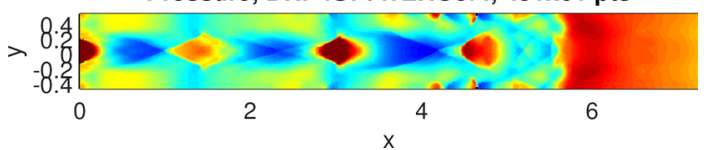

Fig. 15 Inviscid duct flow: pressure contours at time $t=1.32 \mathrm{~ms}$ computed by WENO5 (upper), C06DS+WENO5FI (middle), and DRP4S7+WENO5FI (bottom). Duct grid has $431 \times 61$ grid points
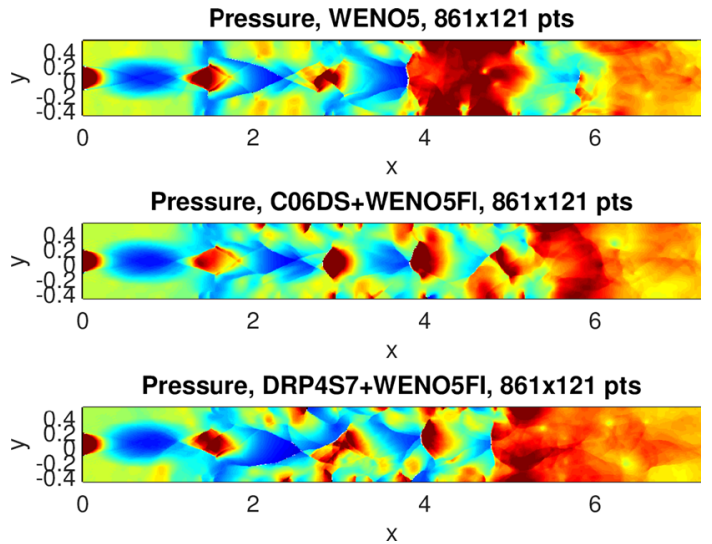

Fig. 16 Inviscid duct flow: pressure contours at time $t=1.32 \mathrm{~ms}$ computed by WENO5 (upper), C06DS+WENO5FI (middle), and DRP4S7+WENO5FI (bottom). Duct grid has $861 \times 121$ grid points

ter uses the wavelet flow sensor to determine the amount of numerical dissipation and location where to apply it; see $[6,16]$. Finally, the lower subplot shows the solution by the nonlinear filter scheme when the fourth-order DRP scheme with a seven-point grid stencil (DRP4S7) is used as the base scheme, DRP4S7DS+WENO5FI. For simplicity of discussion, hereafter the letters DS behind DRP4S7 are not indicated. The nonlinear WENO5FI dissipation is used to filter the solution after each full time step of the Runge-Kutta method.

A three-grid refinement study is shown in Figs. 16 and 17, where the same quantities as in Fig. 15 are plotted, but computed on finer grids. Figure 16 uses $861 \times 121$ grid points in the duct, and Fig. 17 uses $1721 \times 241$ grid points. The flow appears to be unstable, with an increasing number of small scales emerging as the grid is refined.

Figures 18, 19, and 20 show a similar grid refinement study, but at the later time $10.62 \mathrm{~ms}$. This corresponds to around 130, 000 time steps on the $1721 \times 241$ grid. Figure 18
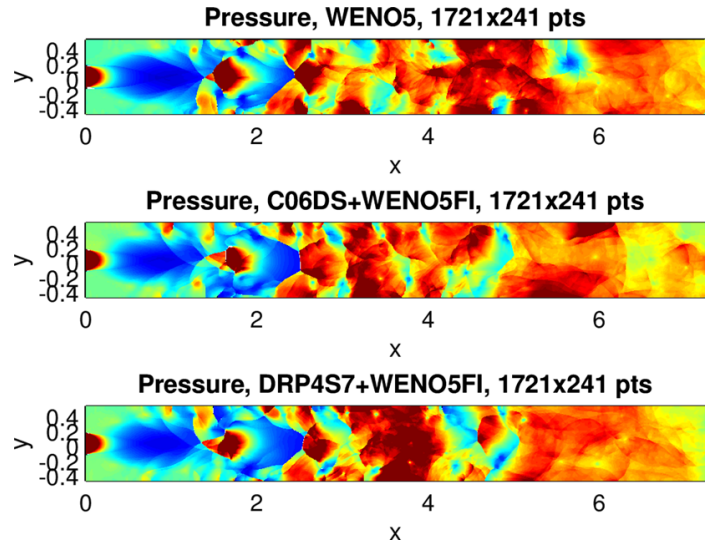

Fig. 17 Inviscid duct flow: pressure contours at time $t=1.32 \mathrm{~ms}$ computed by WENO5 (upper), C06DS+WENO5FI (middle), and DRP4S7+WENO5FI (bottom). Duct grid has $1721 \times 241$ grid points

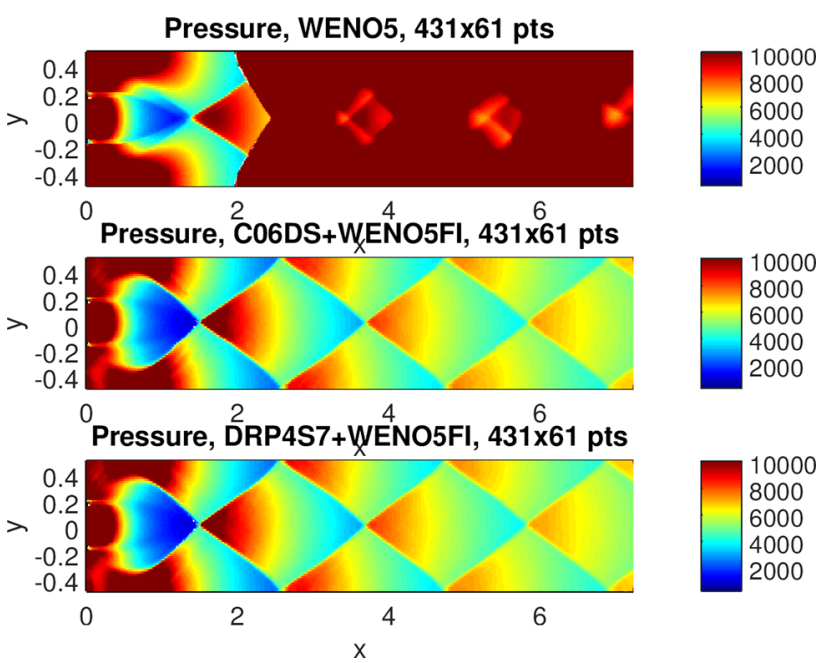

Fig. 18 Inviscid duct flow: pressure at time $t=10.62 \mathrm{~ms}$ computed by WENO5 (upper), C06DS+WENO5FI (middle), and DRP4S7+WENO5FI (bottom). Duct grid has $431 \times 61$ grid points

displays results with $431 \times 61$ points in the duct grid. A more stable shock structure appears in the finer grid simulations shown in Figs. 19 and 20 using $861 \times 121$ and $1721 \times 241$ grid points.

A detailed study of the differences is obtained by plotting the pressure vs. $y$ for grid lines $x=$ constant, i.e., across the duct. This is done as shown in Fig. 21 for $x=0.69$ (top), $x=2.72$ (middle), and $x=3.98$ (bottom). Four different numerical schemes are compared (C06DS+WENO5FI, C06+WENO5FI, DRP4S7+WENO5FI, and WENO5).

Figure 22 shows pressure versus time at $x=3.615$ on the upper wall of the duct on the finest grid. After the initial transient, the pressure oscillates around a constant level. The noise is higher in the WENO5 computation, possibly due to differences in the numerical implementation of the boundary conditions. 


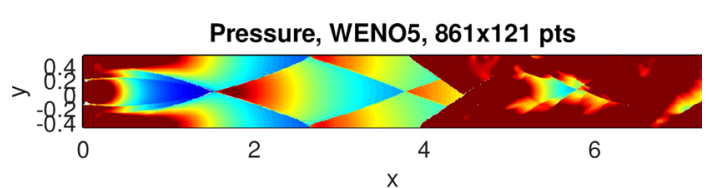

Pressure, C06DS+WENO5FI, $861 \times 121$ pts

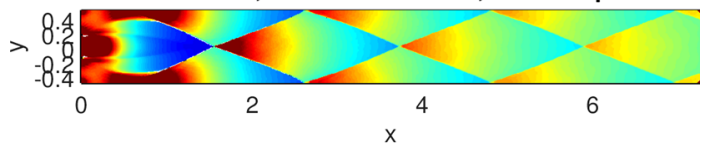

Pressure, DRP4S7+WENO5FI, 861×121 pts

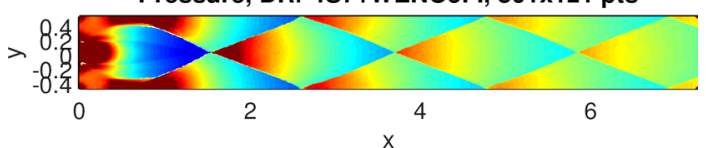

Fig. 19 Inviscid duct flow: pressure at time $t=10.62 \mathrm{~ms}$ computed by WENO5 (upper), C06DS+WENO5FI (middle), and DRP4S7+WENO5FI (bottom). Duct grid has $861 \times 121$ grid points
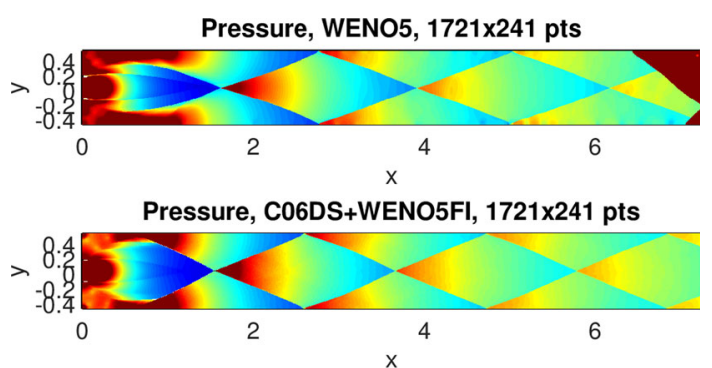

Pressure, DRP4S7+WENO5FI, 1721×241 pts

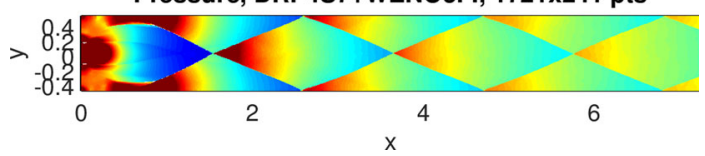

Fig. 20 Inviscid duct flow: pressure at time $t=10.62 \mathrm{~ms}$ computed by WENO5 (upper), C06DS+WENO5FI (middle), and DRP4S7+WENO5FI (bottom). Duct grid has $1721 \times 241$ grid points

Since the grid refinement of the three $y$-cross sections at $t=10.62 \mathrm{~ms}$ appeared to require a longer-time integration before we can judge whether a symmetric steady pattern emerges, we restarted the computations and recorded the same comparison at double the end time at $t=21.24 \mathrm{~ms}$. See Figs. 23, 24, 25, and 26. Figure 24 shows a pressure comparison at time $t=21.24 \mathrm{~ms}$ using $1721 \times 241$ grid points. WENO5 exhibits a more symmetric steady-like flow near the exit with the finest grid and double the time integration than the one for $t=10.62 \mathrm{~ms}$. For the medium grid, WENO5 still has not settled to a symmetric steady-like flow.

By comparing Fig. 26 with Fig. 25 at the same end time $t=$ $21.24 \mathrm{~ms}$, it is evidenced that using $861 \times 121$ and $1721 \times 241$ grid points is approaching nearly grid convergent symmetriclike steady patterns. WENO5 exhibits very different pressure values at the three cross sections than the rest of the methods.
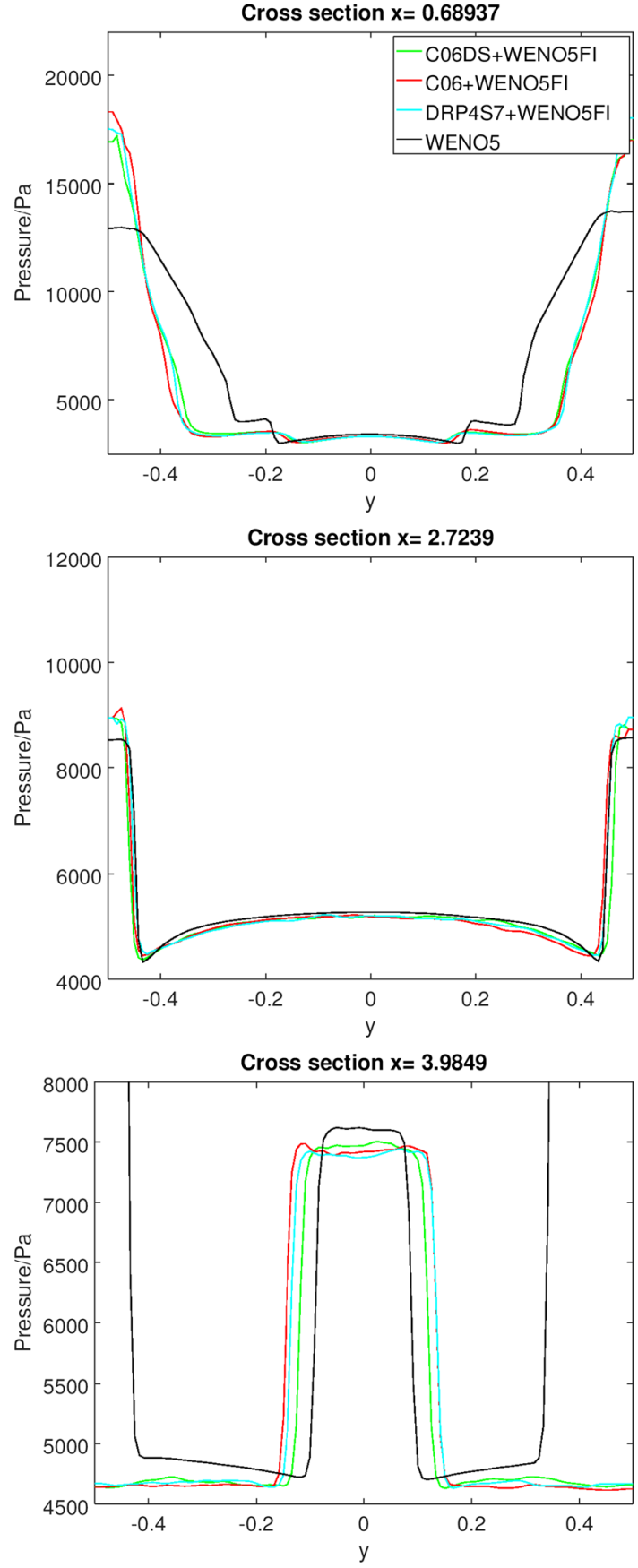

Fig. 21 Inviscid duct flow: pressure versus $y$ for $x=0.69$ (top), $x=2.72$ (middle), and $x=3.98$ (bottom). Comparison of several schemes, all using $861 \times 121$ grid points at $t=10.62 \mathrm{~ms}$

\section{Concluding remarks}

Several skew-symmetric versions of the high-order centralbased schemes, in conjunction with adaptive low-dissipation control via a nonlinear filter step to aid with stability and accuracy simulations, are explored for CAA turbulence applications. The central DRP (dispersion relation preserving) schemes as well as classical central schemes of arbitrary 


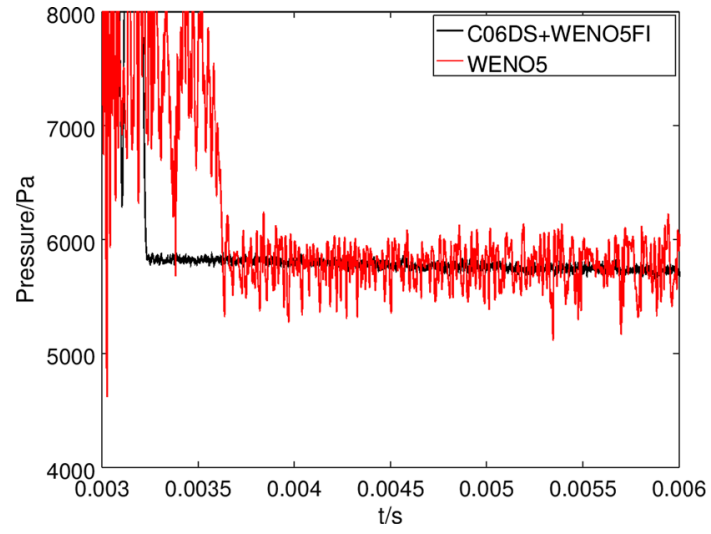

Fig. 22 Inviscid duct flow: pressure versus time at the upper wall of the duct. Comparison of two methods, WENO5 (red) and C06DS+WENO5FI (black), using $1721 \times 241$ grid points
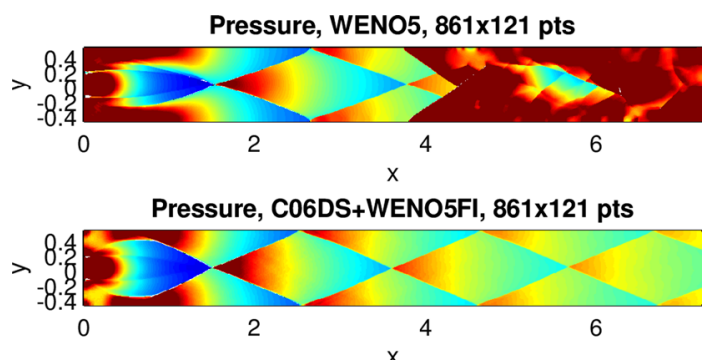

Pressure, DRP4S7+WENO5FI, 861x121 pts

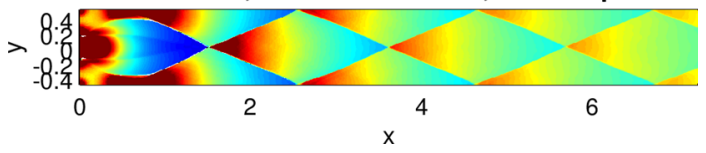

Fig. 23 Inviscid duct flow: pressure at time $t=21.24 \mathrm{~ms}$ computed by WENO5 (upper), C06DS+WENO5FI (middle), and DRP4S7+WENO5FI (bottom). Duct grid has $861 \times 121$ grid points at $t=21.24 \mathrm{~ms}$. The integration time is twice the duration as in Fig. 21

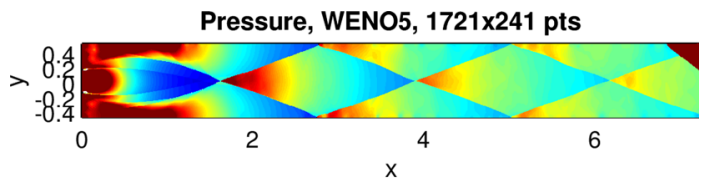

Pressure, C06DS+WENO5FI, 1721x241 pts
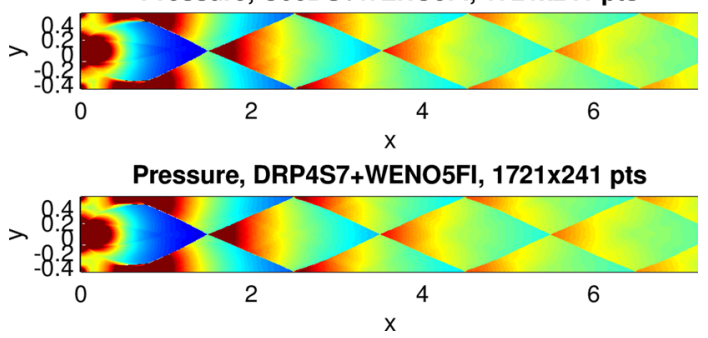

Fig. 24 Inviscid duct flow: pressure at time $t=21.24 \mathrm{~ms}$ computed by WENO5 (upper), C06DS+WENO5FI (middle), and DRP4S7+WENO5FI (bottom). Duct grid has $1721 \times 241$ grid points at $t=21.24 \mathrm{~ms}$. The integration time is twice the duration as in Fig. 21
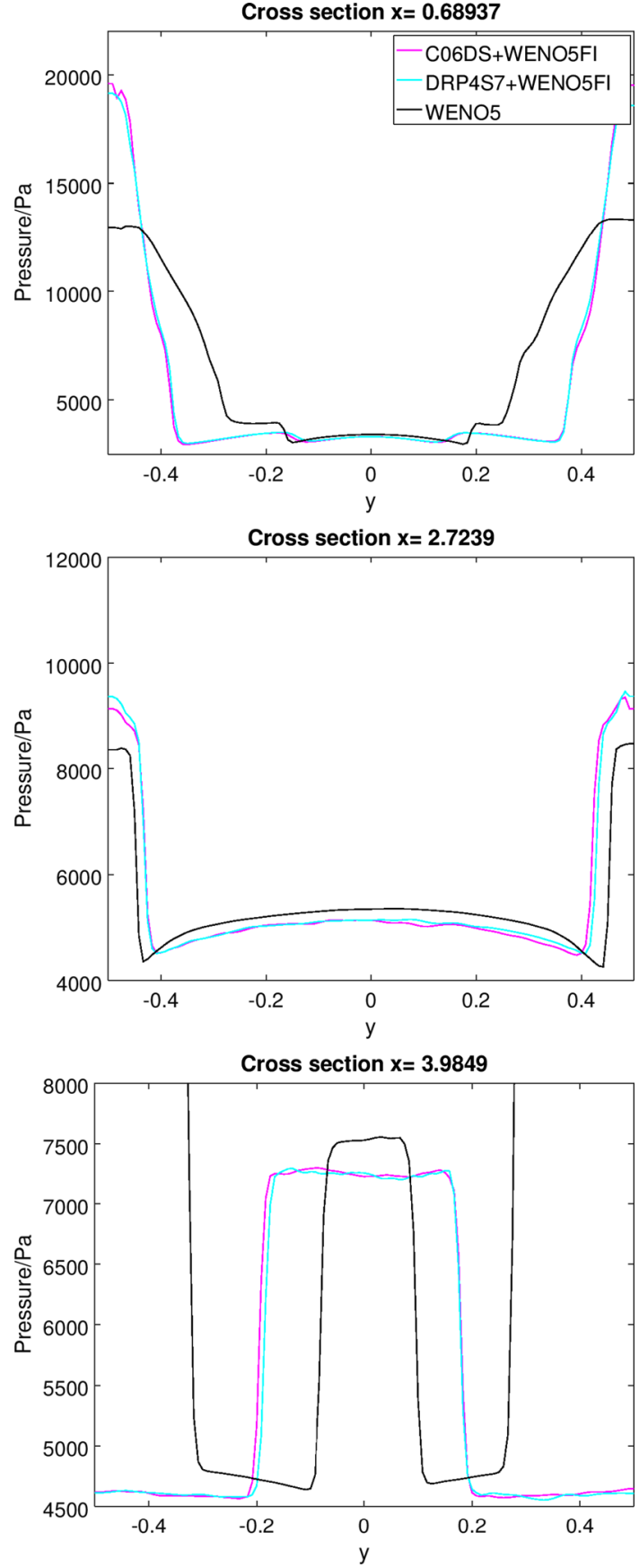

Fig. 25 Inviscid duct flow: pressure versus $y$ for $x=0.69$ (top), $x=2.72$ (middle), and $x=3.98$ (bottom). Comparison of several schemes, all using $861 \times 121$ grid points at $t=21.24 \mathrm{~ms}$. The integration time is twice the duration as in Fig. 21

orders fit into the framework of skew-symmetric splitting of the inviscid flux derivatives. Preliminary studies indicated that the nonlinear filter schemes of Yee et al., Yee and Sjögreen, and Kotov et al. [5,7-10,16] are suitable for CAA turbulent computations and provide accurate and stable numerical results. 

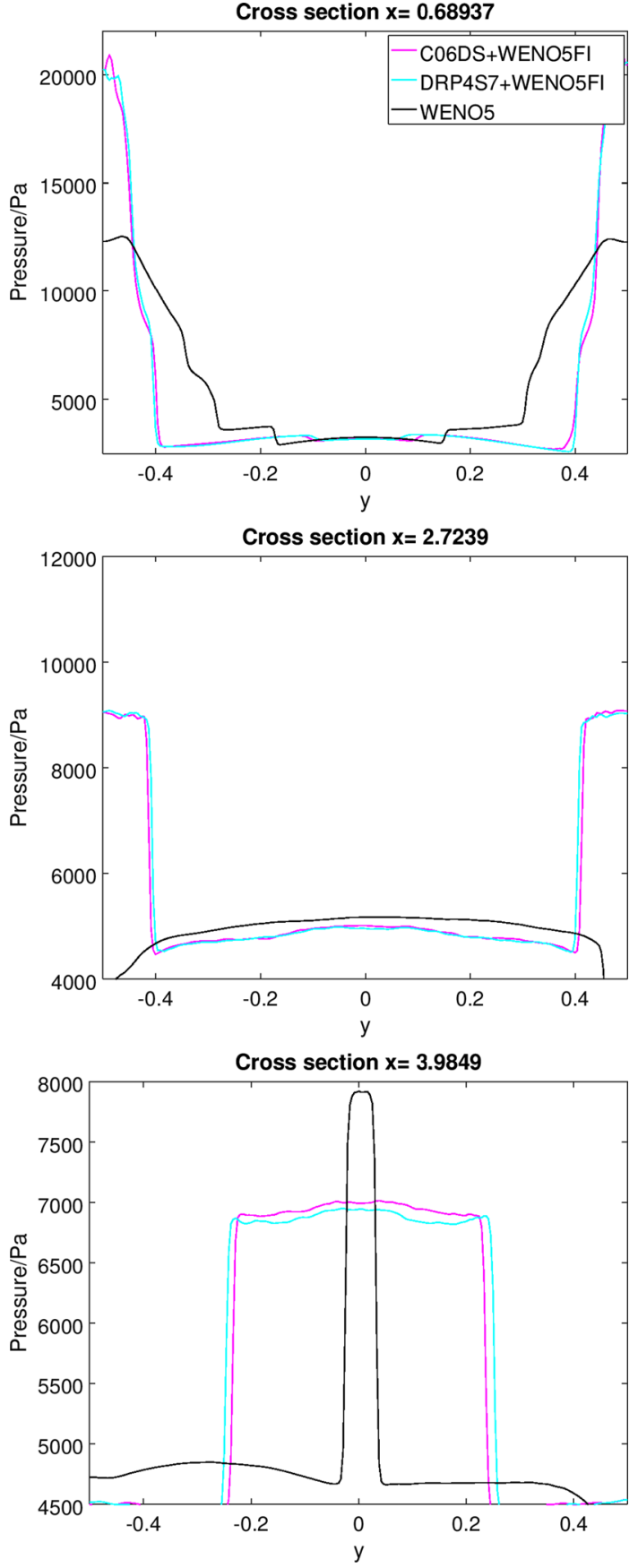

Fig. 26 Inviscid duct flow: pressure versus $y$ for $x=0.69$ (left), $x=2.72$ (middle), and $x=3.98$ (right). Comparison of several schemes, all using $1721 \times 241$ grid points at $t=21.24 \mathrm{~ms}$. The integration time is twice the duration as in Fig. 21

The current study of the duct flow is a step toward solving more realistic CAA engineering problems than previously done with the nonlinear filter scheme. In such problems, the flow interacts strongly with the boundaries of the domain, grid refinement interfaces are present, and Reynolds numbers can be very high. Future work will be to test, and improve as needed, the nonlinear filter schemes for these types of simulations.

Specifically, we plan to analyze in more detail the reasons for the differences in results between WENO and centered schemes for the duct flow problem. Furthermore, we plan to use the Navier-Stokes equations and to add fine grids to resolve the boundary-layer regions. For the duct flow problem, this will allow us to study the behavior of the flow physics under different pressure ratios, $\tau$.

Acknowledgements Funding was provided by Ames Research Center (Grant No. 1009492.02.01.01.05.02).

\section{References}

1. Martín, M.P., Taylor, E.M., Wu, M., Weirs, V.G.: A bandwidthoptimized WENO scheme for the effective direct numerical simulation of compressible turbulence. J. Comput. Phys. 220, 270-289 (2006). https://doi.org/10.1016/j.jcp.2006.05.009

2. Fu, L., Hu, X.Y., Adams, N.A.: A family of high-order targeted ENO schemes for compressible-fluid simulations. J. Comput. Phys. 305, 333-359 (2016). https://doi.org/10.1016/j.jcp.2015.10.037

3. Subramaniam, A., Wong, M.L., Lele, S.K.: A high-order and highresolution nonlinear weighted compact scheme for compressible flows involving shocks and turbulence. 23rd AIAA Computational Fluid Dynamics Conference, Denver, CO, AIAA Paper 2017-4108 (2017). https://doi.org/10.2514/6.2017-4108

4. Pirozzoli, S.: Numerical methods for high-speed flows. Ann. Rev. Fluid Mech. 43(1), 163-194 (2011). https://doi.org/10.1146/ annurev-fluid-122109-160718

5. Yee, H.C., Sandham, N.D., Djomehri, M.J.: Low-dissipative highorder shock-capturing methods using characteristic-based filters. J. Comput. Phys. 150(1), 199-238 (1999). https://doi.org/10.1006/ jcph.1998.6177

6. Sjögreen, B., Yee, H.C.: Multiresolution wavelet based adaptive numerical dissipation control for high order methods. J. Sci. Comput. 20(2), 211-255 (2004). https://doi.org/10.1023/b:jomp. 0000008721.30071.e4

7. Yee, H.C., Sjögreen, B.: Development of low dissipative high order filter schemes for multiscale Navier-Stokes/MHD systems. J. Comput. Phys. 225, 910-934 (2007). https://doi.org/10.1016/j. jcp.2007.01.012

8. Kotov, D.V., Yee, H.C., Wray, A.A., Hadjadj, A., Sjögreen, B.: High order numerical methods for the dynamic SGS model of turbulent flows with shocks. Commun. Comput. Phys. 19, 273-300 (2016). https://doi.org/10.4208/cicp.211014.040915a

9. Kotov, D.V., Yee, H.C., Wray, A.A., Sjögreen, B., Kritsuk, A.G.: Numerical dissipation control in high order shock-capturing schemes for LES of low speed flows. J. Comput. Phys. 307, 189202 (2016). https://doi.org/10.1016/j.jcp.2015.11.029

10. Yee, H.C., Vinokur, M., Djomehri, M.J.: Entropy splitting and numerical dissipation. J. Comput. Phys. 162(1), 33-81 (2000). https://doi.org/10.1006/jcph.2000.6517

11. Sjögreen, B., Yee, H.C., Kotov, D.: Skew-symmetric splitting and stability of high order central schemes. J. Phys.: Conf. Ser. 837, 012019 (2017). https://doi.org/10.1088/1742-6596/837/1/012019

12. Sjögreen, B., Yee, H.C.: Skew-symmetric splitting for multiscale gas dynamics and MHD turbulence flows. J. Sci. Comput. (2018)

13. Sjögreen, B., Yee, H.C.: On high order entropy conservative numerical flux for multiscale gas dynamics and MHD simulations. Spectral and High Order Methods for Partial Differential Equations 
ICOSAHOM 2016, pp. 407-421. Springer, Cham (2017). https:// doi.org/10.1007/978-3-319-65870-4_29

14. Sjögreen, B., Yee, H.C.: High order entropy conservative central schemes for wide ranges of compressible gas dynamics and MHD flows. J. Comput. Phys. 364, 153-185 (2018). https://doi.org/10. 1016/j.jcp.2018.02.003

15. Sjögreen, B., Yee, H.C.: Accuracy consideration by DRP schemes for DNS and LES of compressible flow computations. Comput. Fluids 159, 123-136 (2017). https://doi.org/10.1016/j.compfluid. 2017.09.017

16. Yee, H.C., Sjögreen, B.: High order filter methods for wide range of compressible flow speeds. In: Lecture Notes in Computational Science and Engineering, vol. 76, pp. 327-337. Springer, Berlin (2011). https://doi.org/10.1007/978-3-642-15337-2_30

17. Harten, A.: The artificial compression method for computation of shocks and contact discontinuities: III. Self-adjusting hybrid schemes. Math. Comp. 32, 363-389 (1978). https://doi.org/10. 1090/S0025-5718-1978-0489360-X

18. Arakawa, A.: Computational design for long-term numerical integration of the equations of fluid motion: Two-dimensional incompressible flow. Part I. J. Comput. Phys. 1, 119-143 (1966). https:// doi.org/10.1016/0021-9991(66)90015-5

19. Blaisdell, G.A., Spyropoulos, E.T., Qin, J.H.: The effect of the formulation of nonlinear terms on aliasing errors in spectral methods. Appl. Number. Math. 21(3), 207-219 (1996). https://doi.org/10. 1016/0168-9274(96)00005-0

20. Ducros, F., Laporte, F., Soulères, T., Guinot, V., Moinat, P., Caruelle, B.: High-order fluxes for conservative skew-symmetric-like schemes in structured meshes: Application to compressible flows. J. Comput. Phys. 161, 114-139 (2000). https://doi.org/10.1006/ jcph.2000.6492

21. Sjögreen, B., Yee, H.C.: On skew-symmetric splitting and entropy conservation schemes for the Euler equations. In: Numerical Mathematics and Advanced Applications 2009, pp. 817-827. Springer, Cham (2010). https://doi.org/10.1007/978-3-642-11795-4_88

22. Sandham, N.D., Li, Q., Yee, H.C.: Entropy splitting for high-order numerical simulation of compressible turbulence. J. Comput. Phys. 178, 307-322 (2002). https://doi.org/10.1006/jcph.2002.7022

23. Emmert, T., Lafon, P., Bailly, C.: Numerical study of self-induced transonic flow oscillations behind a sudden duct enlargement. Phys. Fluids 21(10), 106105 (2009). https://doi.org/10.1063/1.3247158

24. Yee, H.C., Sjögreen, B.: Recent developments in accuracy and stability improvement of nonlinear filter methods for DNS and LES of compressible flows. Comput. Fluids 169, 331-348 (2018). https:// doi.org/10.1016/j.compfluid.2017.08.028

25. Yee, H.C., Sjögreen, B.: Adaptive filtering and limiting in compact high order methods for multiscale gas dynamics and MHD systems. Comput. Fluids 37(5), 593-619 (2008). https://doi.org/10.1016/j. compfluid.2007.07.015
26. Kennedy, C.A., Gruber, A.: Reduced aliasing formulations of the convective terms within the Navier-Stokes equations for a compressible fluid. J. Comput. Phys. 227(3), 1676-1700 (2008). https:// doi.org/10.1016/j.jcp.2007.09.020

27. Pirozzoli, S.: Generalized conservative approximations of split convective derivative operators. J. Comput. Phys. 229(19), 71807190 (2010). https://doi.org/10.1016/j.jcp.2010.06.006

28. Sjögreen, B., Yee, H.C.: Two Decades Old Entropy Stable Method for the Euler Equations Revisited. Proceedings of the ICOSAHOM-2018, July 9-13, London, UK (2018)

29. Tam, C.K.W.: A CAA primer for practicing engineers. Technical Report AEDC-TR-08-2, Arnold Engineering Development Center, Arnold Air Force Base (2008)

30. De Roeck, W., Desmet, W., Baelmans, M., Sas, P.: An overview of high-order finite difference schemes for computational aeroacoustics. Proceedings of the International Conference on Noise and Vibration Engineering, pp. 353-368 (2004)

31. Tam, C.K.W.: Computational Aeroacoustics: A Wave Number Approach. Cambridge Aerospace Series. Cambridge University Press, Cambridge (2012). https://doi.org/10.1017/ CBO9780511802065

32. Brambley, E.J.: Optimized finite-difference (DRP) schemes perform poorly for decaying or growing oscillations. J. Comput. Phys. 324, 258-274 (2016). https://doi.org/10.1016/j.jcp.2016.08.003

33. Haras, Z., Ta' asan, S.: Finite difference schemes for long-time integration. J. Comput. Phys. 114, 265-279 (1994). https://doi.org/10. 1006/jcph.1994.1165

34. Yee, H.C., Klopfer, G.H., Montagné, J.-L.: High-resolution shockcapturing schemes for inviscid and viscous hypersonic flows. J. Comput. Phys. 88(1), 31-61 (1990). https://doi.org/10.1016/00219991(90)90241-R

35. Kotov, D.V., Yee, H.C., Panesi, M., Prabhu, D.K., Wray, A.A.: Computational challenges for simulations related to the NASA electric arc shock tube (EAST) experiments. J. Comput. Phys. 269, 215-233 (2014). https://doi.org/10.1016/j.jcp.2014.03.021

36. Zhang, S., Jiang, S., Zhang, Y.-T., Shu, C.-W.: The mechanism of sound generation in the interaction between a shock wave and two counter-rotating vortices. Phys. Fluids 21(7), 076101 (2009). https://doi.org/10.1063/1.3176473

Publisher's Note Springer Nature remains neutral with regard to jurisdictional claims in published maps and institutional affiliations. 